\title{
Modern matrons and infection control practices: aspirations and realities
}

\section{Nelya Koteyko and Brigitte Nerlich}

\begin{abstract}
Modern matrons were introduced in 2001 by the Department of Health to lead clinical teams in the prevention of healthcare-associated infection. The facilitative role of modern matron requires both managerial and entrepreneurial skills and senior nurses are expected to lead by example, inspire, motivate and empower others, and thus to conform to the 'transformational leadership' style that foregrounds the importance of interpersonal and influencing skills. In this paper we identify problems that challenge this model of the modern matron and link them to possible problems in infection control. The study describes cases of difficulty in fulfilling leadership requirements because of organizational barriers to empowerment despite arguments to the contrary. Unless a significant budgetary responsibility is made part of the modern matron role, personal skills (communication, problem solving) alone may not be sufficient to sustain it and may not lead to achieving control over infection which was the initial trigger for instituting this role.
\end{abstract}

Keywords: modern matron, transformational leadership, discourse analysis.

\section{Introduction}

MRSA infections have been on the rise since the 1990s. The reasons for this are not clear, but contributing factors may include the emergence of the epidemic strains EMRSA-15 and EMRSA-16, failure to introduce and maintain suitable infection control procedures, particularly handwashing, increases in movement of patients, visitors and staff, inadequate ward staffing levels, inadequate isolation facilities, high bed occupancy rates, and overall poor hospital cleanliness (see Enright, 2005; Grundmann et al, 2002 for an overview).

'Modern matrons' were created in response to public demand for 'an authoritative figure who would not only provide clinical leadership, but who would also be easily identifiable, have close contact with patients and ensure delivery of care to 
the highest standards' - ensuring prevention of hospital-acquired infection. As it is uncertain how the authoritative management style of matrons in the past fits into today's nursing and healthcare culture and the focus on empowerment (Oughtibridge, 2003), it was suggested that the role could be seen as enabling, rather than strictly authoritarian, using transformational leadership styles (Bryman, 1992).

In this paper we will identify problems that challenge this model of the modern matron and link them to possible problems in infection control - in other words, look at gaps between aspirations and realities which may lead to difficulties in preventing and controlling infection. Drawing on policy documents and interview data, we aim to explore the impact of the 'transformational leadership' style in the role of modern matron with regards to infection control practices, and to highlight experiences of modern matrons which can instruct future guidance for an increased involvement of matrons in infection control.

\section{'Transformational leadership' and modern matron role}

The Royal College of Nursing places an emphasis on senior nurses as 'transformational leaders' (RCN, 2004, 2005) who are expected to lead clinical teams in the prevention of healthcare-associated infection such as MRSA and, more recently, Clostridium difficile. There is a vast literature on leadership across the management, psychological and sociological sciences. Recent work is dominated by research on the concept of transformational leadership (Bass and Avolio, 1993) that foregrounds the importance of interpersonal and influencing skills. The effective 'transformational' leader has the skill to motivate, inspire, stimulate and facilitate others (Kouzes and Posner, 1997). Despite problems with the application of this model in other public sector organizations (Currie et al, 2005) and critical comments that management in the NHS now bears 'no resemblance to the consensual management arrangements in which matrons operated in the 1970s' (Bolton, 2003: 123), evidence is said to accumulate for the effectiveness of transformational leadership skills in fostering performance in healthcare (Clegg, 2000, see also RCN, 2004: 164).

\section{Data}


Two sets of texts were collected to compare and contrast the role of the modern matron prescribed in policies with realities on the ground: a corpus of Department of Health documents on modern matrons (see References) and a corpus of semistructured interviews conducted in a large healthcare trust in the East Midlands. The first set is made up of 11 policies issued between 2001 and 2006 dealing with the introduction of modern matrons and their involvement in infection control practices. The second set of texts is comprised of 11 interview transcripts with modern matrons. All participants but one had been in post as a modern matron for over a year since the role was introduced within the trust.

The interview structure was designed to be as informal and open-ended as possible. Below are some examples of the questions used as prompts:

- Please describe your role in the hospital?

- What are the main challenges that you face?

- Could you describe your typical day?

- Has MRSA affected your work in any way?

\section{Ethical considerations}

The study was approved by the Local Research Ethics Committees for the whole trust. Written consent was obtained from all senior nurses after they had been given information indicating the purpose of the study and information about how the data would be used. Assurances of anonymity and confidentiality were given.

\section{Methodology}

Methods of corpus linguistics and discourse analysis were used to identify main themes emerging in policies and interviews. Whereas discourse analysts study language use in social contexts on the basis of a relatively small number of texts, corpus linguists advocate an analysis of language based on large collections of authentic texts - corpora (Sinclair, 1991). In this study we used concordances and word lists - computational tools developed for processing large volumes of real language data in order to supplement our qualitative study of discourse. A set of concordance lines presents instances of a word or phrase in the centre, with words that come before and after it to the left and right (see examples below) and provides a quick access to contexts where a selected word is used (Skelton and Hobbs, 1999). Lists of words sorted according to their frequency (see Figure 1 for an example) allow precise quantification of the most commonly 
used words and can be employed to establish the 'aboutness' of individual texts and whole corpora.

First, word frequencies were calculated with the help of WordSmith software (Scott, 1999). Concordances were then generated for high and medium frequency words to explore their contexts of use. After the examination of their concordances, frequent words were grouped according to semantic criteria (for example, words such as talk, speak and say were assigned to a subset of 'communication'). This allowed us to retrieve words employed to prescribe responsibilities of the modern matron in the policies and compare them to words used by modern matrons themselves to describe their visions of their role and responsibilities.

For an in-depth study of the main themes identified in the matrons' accounts with the help of the corpus linguistic techniques (Koteyko and Carter, in press) we chose the concept of 'role analysis' introduced by the sociologist Erving Goffman. According to Goffman social actors do not passively enact a role. An actor can not only 'embrace' a role, i.e. completely disappear into the self available in the situation, but also be emotionally detached and in this way keep 'role distance' (Goffman, 1961, see also Bolton, 2005). The concept of role distance provides a sociological means of dealing with one type of divergence between obligation and actual performance. This is especially useful for our study when considering how senior nurses can be both skeptical and enthusiastic in their enactment of the Department of Health allocated role of the modern matron rather then blindly following the 'powerful discourse of enterprise' (du Gay, 1996).

\section{Modern matrons and infection control practices in policies}

Word lists generated for the corpus of policies reveal several thematic trends. The following words which appear towards the top of the word list (arranged in the descending order of frequency) reflect the focus on customer care language: lead, standards, cleanliness, authority, enable, skills, power, and visible and paint a picture of a 'thoroughly modern matron': a hybrid manager in a complex organization who can provide 'visible assurance that there is an authoritative figure [...] able to intervene where necessary to resolve problems quickly and effectively' (DH, 2001). 
Concordances drawn for these words reveal a rich rhetoric of 'empowerment' around the modern matron role (Appendix 1.1). The visibility aspect is also consistently stressed throughout the policies (Appendix 1.2). The Health Service Circular on Matrons (2001), for example, links visibility and accountability to the image of the old style matron - 'an authoritative figure who is clearly 'in-charge' of standards'. Ward sisters and unit managers are to be given 'authority and support they need to resolve clinical issues and to ensure that the basics of care are right' (DoH, 2001: 6).

But to what extent do the postholders identify themselves with this role of an empowered 'agent for change'? Below we examine the matrons' accounts to see how far the rhetoric of 'empowerment' reaches into the day-to-day realities of infection control practices.

\section{Modern matron role and infection control practices in the interviews}

The study of word lists offers a good opportunity to take a preliminary look at the thematic composition of interviews. Transcripts were processed to remove the interviewer's words so that only respondents' words remained and the remaining text was used for analysis. Frequent content words ${ }^{1}$ (see Fig. 1) were arranged into the following broad groups: 'health care' (nurse, care, ward, etc.); 'organisation and administration' (work, environment, services, manager, standards), 'communication' (talk, say, tell, discuss), and 'difficulties' (issues, need, try, difficult).

Figure 1 should appear about here

Concordances of words from the group 'organisation and administration' show that matrons appear to have internalised much of the policy terminology, particularly the vocabulary of 'quality care', as they speak about 'clinical leadership' 'care for the environment', 'patient journey',

\footnotetext{
${ }^{1}$ Content words carry lexical meaning and include nouns, verbs, adjectives, and most adverbs, whereas function or grammatical words serve to express grammatical relationships with other words in a sentence. In our analysis, the grammatical classes of prepositions, pronouns and adverbs were removed from the word lists.
} 
'patient experience', 'improvement', etc. when describing their role and involvement in infection control practices. However, concordances of words belonging to the group of 'difficulties' helped us to identify examples where matrons appear to disassociate themselves from the role of 'an empowered manager' who can resolve problems in infection control efficiently.

For example, concordances of need (see Fig. 1), try (Appendix 2.1), and issue (Appendix 2.3) were used as a quick guide to difficulties experienced by modern matrons in the day-to-day enactment of their role. One of the matrons, for example, used need to refer to the pressures that result from the conflict between obligations and realities of such a complex negotiated order as a hospital.

So for instance for one of my areas I need to close twelve beds but I need to do the same level of, we need to do the same level of activity but I potentially need to lose some nurses as well. So we've got to do the same amount of work in less beds with less staff (interview 9).

An act of 'role distancing' could be observed when a matron referred to the numerous meetings she had to attend as 'the bane of my life' (with a comic sigh). She then continued to explain:

I mean if you looked at my diary there would be all these meetings and me running around different places in between them trying to actually do the parts of my job that I felt were important like being visible, talking to the staff on the shop floor, dealing with complaints.(interview 21)

The high frequency of lots of/a lot of alerted us to the multiplicity of tasks that modern matrons are expected to perform on the everyday basis. Words following lots of/a lot of, such as managing, emails, audits, constraints, etc. (Appendix 2.2) denote a number of things that may receive precedence over infection control related issues.

[...] I would do lots of HR type issues, lots and lots of those kind of things, I do sickness interviews, recruitment, general sort of disciplinary type, performance management kind of things. I'm very 
involved at the moment in meetings around workforce change. I meet regularly with my business manager, my divisional nurse, my finance link person. (interview 12)

The study of 'try/trying' illustrated the lack of real power to bring about changes in practices and procedures. The following quote betrays a certain deal of scepticism about the responsibilities of the modern matron with regard to patient care and infection control:

And I think that is down to policy, locally and nationally really in terms of cuts, savings, making people responsible for things and accountable for things that they actually have no jurisdiction over. It's just bonkers really you know. So like I mean they're the key things and it's just working it's just working away trying to, trying to do the right thing really. (interview 9)

Modern matrons were given power to withhold payment for contracted cleaning, but this does not seem to have significantly influenced their sense of 'empowerment' in dealing with cleaners:

I do think that we should have more, more control over the domestic services and what, and be involved in decision making when they reduce their numbers or have sickness (interview 12 )

This type of 'empowerment' might therefore have been more symbolic than real:

I'd like to be given a fair chance to deliver what's expected of me and that's to deliver a good quality patient service, reducing the risks to patients, including infection. And whilst I'm being told that I can't recruit to nursing vacancies and I'm having to cut beds and I'm having to reduce staff and I know that all the other areas are and I have actually no responsibility, they tell me I have responsibility for the cleanliness in my areas but I don't have any input over the staff that provide that service. (interview 9)

The same matron also pointed out what can be called her 'powerlessness' when describing her experience of trying to discuss issues around cleaning. In this 
case, communication skills - one of the attributes of the transformational leadership style- seem to have little effect:

He didn't feel that he could answer those questions and thought I should talk to this person, so I talked to that person who felt it should be that person. Which again is a bit like the cleaning thing, you know I can talk to the domestics and say look guys can you just make sure that you give the side rooms a good clean out because they're you know. But then their boss can come along and say right you've done that bit now, you need to move to another area (interview 9).

Another barrier to achieving improvements in infection control and patient services was raised in the interviews. This was the question of the relationship with other professions. Improvement of infection control practices such as handwashing and environmental cleanliness requires the modern matron to challenge ways of inter-professional working and organizational culture:

I don't have control over medical staff and that can be challenging sometimes [...]. And it's quite difficult to change habits and perceptions and ideas when it's not my staff. (interview 12)

The visibility aspect of the modern matron role is particularly hard to maintain and indicates 'role diffusion' which stands in the way of the complete 'role embracement' by senior nurses. The diffusion of role takes place both hierarchically as well as geographically. Some senior nurses are expected to coordinate a number of wards across an extensive area:

I cover five wards across the north of the county, so I cover wards that are located right in the north of the county and it means that 40 odd miles between each hospital ward that I cover so that makes my job incredibly difficult in the sense that the visibility aspect of it is almost impossible to maintain. (interview 21)

For others, the hierarchical spread of the role and proliferation of tasks seem to pose a more serious problem as far as the visibility is concerned. With so many responsibilities and different priorities to juggle, the modern matron simply does not have time to be on wards to ensure 'visible presence', which runs contrary to 
the generalised assertion that 'Matron's charter Nurses know what works on the wards' (DH, 2004a). As a result, they have to spend most of their time in the office going through paperwork and overseeing the cleanliness of wards through audits.

I think, I believe the ward sister is responsible for maintaining the levels of cleanliness etc on the ward. I will come in if the audits show that targets aren't being met etc. But because I'm not on the ward 24 hours a day I see that clearly as the ward sister's role. But I'm ultimately responsible. (interview 4)

Diffusion of the role is also evident when matrons talk about their attempts to conform to the expectations and act as a liaison person. On the one hand the matron is supposed to be the 'visible' link between various organisational levels, but on the other this visibility is diluted in so far as it is impossible to be (seen) in various places at once:

I'm perhaps less a clinician and more, and I'm not, and I wouldn't call myself a manager but I sit uncomfortably somewhere between the two. [...] I mean I try to be somewhere between what I call up there which is management and kind of organisational structures and the shop floor, so I see myself as an in-between person. [...] (interview 21)

The matrons' accounts suggest that the impact could have been greater if the 'aspirational' aspects of the role were not as far removed from 'reality' as they seem to be - a gap that should be closely monitored in order not to let failings, especially with regard to infection control, creep in.

\section{Summary}

Data collected from modern matrons indicate that they adopt a pragmatic approach to their responsibilities. Some aspects of their role are welcomed and senior nurses are enthusiastic in their responses to infection control policy initiatives. However, when it comes to such issues of infection control as maintaining cleanliness on the ward and encouraging handwashing by doctors and visitors to prevent cross infection, senior nurses are keen to disassociate 
themselves from the title of 'authority figure' . In these situations, modern matrons tend to see their role as that of a middle/link person restricted by various organizational and financial constraints.

This linking function can, however, become a problem in itself when it comes into conflict with the 'visibility' function of the role and therefore lead to 'role diffusion' and uncertainty, as the modern matron has to negotiate work across both hierarchical and geographical boundaries. A similar problem arises from the conflict between increasing administrative tasks (which make the matron invisible) and wanting to achieve clinical leadership and being accessible to patients.

\section{Conclusions}

The policies call for new ways of working and not a return to old ways of managing staff. One way to move beyond the 'old' style matron role is seen as taking a clinical lead. Here the authority of the modern matron and expertise appear to derive not so much from previous nursing expertise but from transferable skills, such as clinical judgment, observation and communication skills - all attributes of 'transformational leader'.

This study has described cases of difficulty in fulfilling such clinical leadership requirements because of organisational barriers to empowerment despite rhetoric to the contrary. Our analysis of interviews has shown that the modern matron does not achieve the status of a 'powerful figurehead', who would be 'highly visible' on the ward and who would have time and resources to effectively resolve issues in infection control.

Some positional authority is important for building professional credibility, and, subsequently, for introducing and sustaining new initiatives in infection control. As Millward and Bryan (2005: 25) point out in their report on the concept of leadership in healthcare, a clinical leader 'should have some authority over financial and other resources, as well as some ability to upwardly influence health care policy'. Unless a significant budgetary responsibility is made part of the modern matron role, personal skills (communication, problem solving) alone may not be sufficient to sustain it (Hewison, 2001) and may not lead to achieving control over infection which was the initial trigger for instituting this role. 
Achieving a balance between managerial and clinical duties, interpersonal skill, and authority over financial resources remains a major challenge in this crucially important area of health care.

\section{Acknowledgements}

We would like to thank the modern matrons and infection control staff who participated in this research study.

\section{References}

Bass, B. and B. Avolio. (1993). Transformational leadership: a response to critiques. In M. Chemers and R. Ayman (Eds.) Leadership Theory and Research: Perspectives and Directions. New York: Academic Press.

Bolton, S. (2005). Making up managers. Work, Employment \& Society, 19 (1): 523.

Bolton, S. (2003). Multiple Roles: Nurses as Managers in the NHS. International Journal of Public Sector Management, 16 (2) :122-130.

Bryman, A. Charisma and leadership in organizations. Newbury Park CA: Sage, 1992.

Clegg, A. (2000). Leadership: improving the quality of patient care", Nursing Standard, 14 (30): 43-5.

Currie, G., Boyett, I. and Suhomlinova, O. (2005). Transformational leadership in the public sector: A panacea for organisational ills? Public Administration, 83: 265-96.

du Gay, P. (1996). Organizing Identity: Entrepreneurial Governance and Public Management. In S. Hall and P. du Gay (Eds.), Questions of Cultural Identity, London: Sage: 151-69

Enright, M. (2005). MRSA Epidemiology \& Evolution. Science Lecture, 21 April. http://www.brlsi.org/proceed05/lecture0405.htm.

Goffman, E. (1961). Encounters. New York: Bobbs-Merrill.

Grundmann, G., Hori, S., Winter, B., Tami, A. and D. Austin. (2002). Risk factors for the transmission of methicillin-resistant Staphylococcus aureus in an adult intensive care unit: fitting a model to the data, Journal of Infectious Diseases, 185: 481-488.

Hewison, A. (2001). The Modern Matron: reborn or recycled? Journal of Nursing Management, 9: 187-189.

Koteyko, N. and Carter, R. (in press). Discourse of 'transformational leadership'. Health. 
Kouzes, J. and Posner, B. (1997). The Leadership Challenge. San Franscisco, CA: Jossey-Bass.

Millward, L. and K. Bryan. (2005). Clinical Leadership in health care: a position statement. International Journal of Health Care Quality Assurance 18(2), 13-25. Oughtibridge, D. (2003). The modern matron. Nursing Management 10 (2): 2628.

Scott, M. (1999). WordSmith Tools 3. Oxford: Oxford University Press.

Sinclair, J. (1991). Corpus, Concordance, Collocation. Oxford: Oxford University

Press.

Skelton, J. and F. Hobbs. (1999). Concordancing: the use of language-based research in medical communication. The Lancet, 353: 108-111.

\section{Policies}

1. DH. (2001). Modern matrons - strengthening the role of ward sisters and introducing senior sisters (Health Service Circular 2001/010). London: DH.

2. DH. (2002a). Getting Ahead of the Curve: A Strategy for Combating Infectious Diseases. London: DH.

3. DH. (2002b). Modern Matrons in the NHS: A Progress Report. London: DH.

4. DH. (2003a). Modern Matrons - Improving the Patient Experience. London: $\mathrm{DH}$.

5. DH. (2003b). Winning ways-working together to reduce healthcare acquired infection. London: The Stationery Office.

6. DH. (2004a). A Matron's Charter: An action plan for cleaner hospitals. London: $\mathrm{DH}$.

7. DH. (2004b). Towards cleaner hospitals and lower rates of infection. London: $\mathrm{DH}$.

8. RCN. (2004). Evaluation of the modern matron role in a sample of NHS trusts. Final Report. RCN: London.

9. DH. (2005). Saving Lives: a delivery programme to reduce healthcare associated infection including MRSA. London: $\mathrm{DH}$.

10. RCN. (2005). Wipe It Out RCN Campaign on MRSA. RCN: London.

11. DH. (2006). Essential steps to safe, clean care. London: DH.

\section{Appendix 1: Concordances from the corpus of policy documents}




\subsection{Concordances of empower}

Noncordance
1 n to ensure that NHS employers empower appropriately qualified
2 frontline. They are helping to empower nurses and are ensuring
3 e and to inspire, motivate and empower others; the Chief Nursi
4 their leadership skills and to empower other members of their
5 by example and to motivate and empower others. The guidance al
6 and essential aspects of care empower nurses to take on a wi
7 Matrons in the NHS $\quad$. empower nurses - by enabling m
8 eading by example so that they empower nurses to strive for h
9 heir own environment and being empowered to do something about
10 ogether to find solutions...by empowering ward managers and si
11 standards of clinical care and empowering nurses to take on a
12 iven nurses some authority and empowerment and given our patie

\subsection{Concordances of visible}

N Concordance
1 NHS Trust. "Matrons are very visible just by the uniform w
2 ge nurses who provide strong, visible clinical leadership. T
3 ell as cleanliness, should be visible and reliable. Often, p
4 es designed to secure strong, visible clinical leadership ap
5 ng standards and being highly visible to staff, patients and
6 if strong leadership was more visible and closer to the pat
7 ership on wards and be highly visible and accessible to pati
8 ernisation agenda... [and] a visible and accessible figure
9 of standards and being highly visible to staff, patients and
10 thcare associated infection a visible and unambiguous indica
11 bvious to patients. They want visible assurance that there i
12 a group of wards and a highly visible, accessible and author
13 ce; ensuring that matrons are visible, easily identifiable b
14 standards of care providing a visible, accessible and author
15 o that the benefits of highly visible, accessible and author
16 s by providing someone who is visible, works on the wards a
17 me to "walk the floor" and be visible.

\section{Appendix 2: Concordances from the corpus of interviews}

\subsection{Concordances of trying}


1 think when we were actually trying to talk about it the 2 ing people off the beds and trying to restrict visiting

3 king it's just working away trying to, trying to do the 4 ne of the things we've been trying to look at is what I

5 pose in some respects. But trying to maintain this emer

6 ment that you're constantly trying to counter balance an

7 r it could be just that I'm trying to keep myself clinic

8 an acute hospital. So it's trying to look at those sta

9 ently in the throes of just trying to sort that one out

10 they are looking at ways of trying to address that. But

11 a challenge as well you see trying to educate people tha

12 a lot of constraints, we're trying to proactively now ma

\subsection{Concordances of a lot of/lots of}

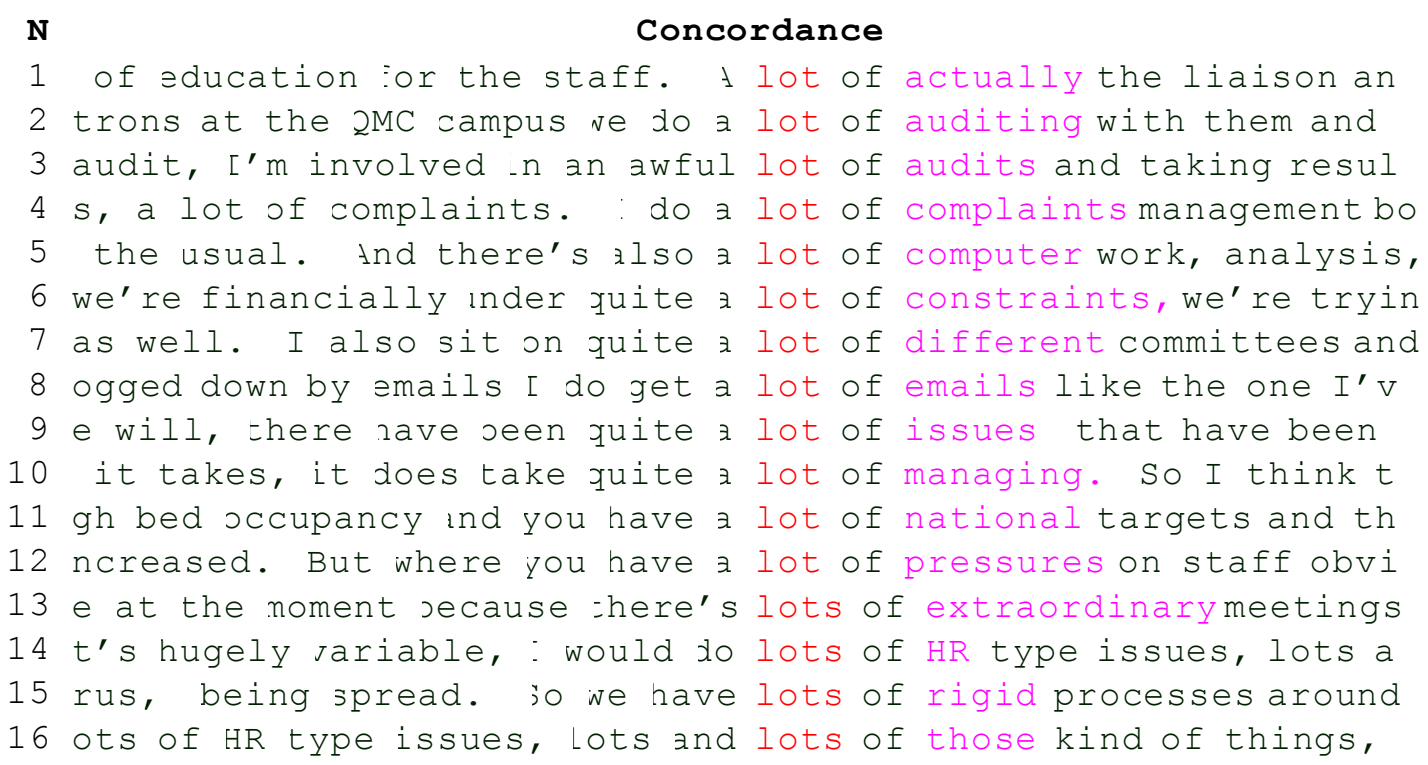

\subsection{Concordances of issues}

\section{$\mathbf{N}$}

1 on and also the cleanlinessissues as well, that's somet

2 et involved -n any alinical issues with patients as requ

3 at there's ro contaminationissues with other patients b

4 e so nany infection zontrol issues going on on that, in

5 looking at capital Eunding issues that that's the one

6 any outstanding laintenanceissues or if something is $n$

7 all the HR, human resource issues within my directorate

8 groups vith auman resource issues to do with monitoring

9 ooking at infection :ontrol issues, looking at environme

10 ts, the service improvementissues, all of those are ba

11 ell. jo any staff sicknessissues, actually recruiting 


\begin{tabular}{|c|c|c|c|c|c|}
\hline Word & Freq. & $\%$ & Word & Freq. & $\%$ \\
\hline KNOW & 514 & 1.81 & DAY & 68 & 0.13 \\
\hline THING & 358 & 0.70 & SEE & 67 & 0.13 \\
\hline THINK & 352 & 0.69 & BED & 64 & 0.13 \\
\hline CAN & 292 & 0.61 & TRY & 63 & 0.12 \\
\hline PATIENT & 268 & 0.53 & THEATRE & 59 & 0.12 \\
\hline ALL & 241 & 0.47 & SERVICE & 58 & 0.11 \\
\hline WARD & 237 & 0.46 & PUT & 57 & 0.11 \\
\hline PEOPLE & 217 & 0.43 & AROUND & 56 & 0.11 \\
\hline GET & 209 & 0.27 & CLEAN & 56 & 0.11 \\
\hline MATRON & 194 & 0.38 & DOMESTIC & 55 & 0.11 \\
\hline INFECTION & 182 & 0.36 & HEALTH & 55 & 0.11 \\
\hline STAFF & 181 & 0.35 & PART & 55 & 0.11 \\
\hline LOT & 179 & 0.35 & ENVIRONMENT & 54 & 0.11 \\
\hline WORK & 171 & 0.34 & SERVICE & 54 & 0.11 \\
\hline MEAN & 161 & 0.32 & NURSING & 53 & 0.10 \\
\hline NURSE & 139 & 0.27 & TEAM & 53 & 0.10 \\
\hline CONTROL & 130 & 0.25 & WAY & 52 & 0.10 \\
\hline MAKE & 115 & 0.23 & TAKE & 51 & 0.10 \\
\hline MRSA & 115 & 0.23 & TERM & 47 & 0.09 \\
\hline HOSPITAL & 108 & 0.21 & ABLE & 46 & 0.09 \\
\hline HAND & 106 & 0.21 & TELL & 45 & 0.09 \\
\hline NEED & 106 & 0.21 & MODERN & 44 & 0.09 \\
\hline LOOK & 105 & 0.21 & STANDARD & 43 & 0.08 \\
\hline GO & 92 & 0.18 & YEAR & 43 & 0.08 \\
\hline COME & 88 & 0.17 & CLEANLINESS & 42 & 0.08 \\
\hline WASH & 82 & 0.16 & ISSUE & 42 & 0.08 \\
\hline MAY & 82 & 0.16 & MEETING & 42 & 0.08 \\
\hline TIME & 80 & 0.16 & CARE & 40 & 0.08 \\
\hline CLEAN & 79 & 0.15 & WANT & 39 & 0.08 \\
\hline GOOD & 79 & 0.15 & DOCTOR & 39 & 0.08 \\
\hline ROLE & 79 & 0.15 & HAPPEN & 38 & 0.07 \\
\hline DIFFERENT & 76 & 0.15 & $\mathrm{JOB}$ & 37 & 0.07 \\
\hline AREA & 75 & 0.15 & KIND & 37 & 0.07 \\
\hline DO & 74 & 0.15 & PRACTICE & 37 & 0.07 \\
\hline SURE & 73 & 0.14 & TRUST & 37 & 0.07 \\
\hline CLINICAL & 72 & 0.14 & EVERY & 36 & 0.07 \\
\hline MANAGE & 72 & 0.14 & HELP & 36 & 0.07 \\
\hline SAY & 71 & 0.14 & MOMENT & 36 & 0.07 \\
\hline AREA & 69 & 0.14 & OWN & 36 & 0.07 \\
\hline BIT & 68 & 0.13 & GENERAL & 37 & 0.07 \\
\hline
\end{tabular}

Figure 1. Word frequencies in the corpus of interview transcripts (cut off point $0,07 \%)$. 